DOI: https://doi.org/10.46296/yc.v4i7edespdic.0072

\title{
ACCIONES PARA LA PREPARACIÓN EN COCINA TRADICIONAL CUBANA EN INSTALACIONES TURÍSTICAS
}

\section{ACTIONS FOR THE PREPARATION OF TRADITIONAL CUBAN CUISINE IN TOURIST FACILITIES}

\author{
Nápoles-Abreu Osvaldo ${ }^{1 *}$; Abascal Obregón Pilar Elena²; Mustelier-Casola Daimara ${ }^{3}$; \\ Crespo Zafra Lourdes Mariana ${ }^{4}$ \\ ${ }^{1}$ Profesor Instructor del departamento Tecnología y Ciencias de los Alimentos, \\ Universidad de Camagüey Ignacio Agramonte Loynaz. Camagüey, Cuba. Orcid: \\ https://orcid.org/0000-0003-0658-4257 \\ 2 Departamento de Alimentos y Bebidas de la Escuela de Hotelería y Turismo de Ciego \\ de Ávila Joaquín de Agüero. Ávila, Cuba. Orcid: https://orcid.org/0000-0002-8615-7812 \\ ${ }^{3}$ Profesora del departamento Tecnología y Ciencias de los Alimentos, Universidad de \\ Camagüey Ignacio Agramonte Loynaz. Camagüey, Cuba. Orcid: https://orcid.org/0000- \\ 0001-5284-5046 \\ ${ }^{4}$ Profesora del departamento Tecnología y Ciencias de los Alimentos, Universidad de \\ Camagüey Ignacio Agramonte Loynaz. Camagüey, Cuba. Orcid: https://orcid.org/0000- \\ 0002-4799-3447
}

*Correo: osvaldo.napoles@reduc.edu.cu

\begin{abstract}
Resumen
El acelerado desarrollo del turismo en la actualidad, demanda recursos humanos altamente preparados para responder a las exigencias de los clientes. Estudios recientes en instalaciones turísticas cubanas han identificado insatisfacciones de los clientes en cuanto a calidad de las elaboraciones culinarias; asociadas a los niveles de preparación del personal de cocina. La presente investigación tuvo como objetivo mostrar los principales resultados en la implementación de acciones para la preparación en cocina tradicional cubana de los especialistas culinarios del Hotel Colonial Cayo Coco, de la provincia de Ciego de Ávila. El trabajo se estructuró en cuatro etapas, empleándose como métodos de investigación: el análisis documental, la observación directa y participativa y la entrevista. La propuesta de acciones de preparación se elaboró tomando en cuenta las deficiencias de los especialistas culinarios, reflejadas en las insatisfacciones de los clientes. Estas acciones fueron validadas por criterio de expertos previamente seleccionados mediante el método Delphi. La implementación de las acciones de preparación contribuyó al incremento cognoscitivo de los especialistas culinarios en temáticas de cocina tradicional cubana y al aumento de la satisfacción de los clientes del Hotel Colonial Cayo Coco, lo cual constituye un instrumento factible para la gestión de la calidad de los servicios de alimentación en instalaciones turísticas del país.
\end{abstract}

Palabras clave: cocina tradicional cubana; preparación profesional; turismo.

\begin{abstract}
The accelerated development of tourism today requires labor force highly trained to respond to customers' demands. Recent studies in Cuban tourist facilities have identified customer dissatisfaction regarding the quality of culinary preparations; associated with the qualification level of the kitchen staff. The objective of this research was to show the main results in the implementation of actions to attend the qualification in traditional Cuban cuisine, of the culinary specialists from the Hotel Colonial Cayo Coco, in Ciego de Avila province. The work was structured in four stages, using as research methods: documentary analysis, direct and
\end{abstract}

Información del manuscrito:

Fecha de recepción: 05 de noviembre de 2020.

Fecha de aceptación: 14 de diciembre de 2020.

Fecha de publicación: 21 de diciembre de 2020. 
participatory observation as well as interviews. The proposal for improving qualification was developed considering the deficiencies of the culinary specialists' training, reflected in the dissatisfaction of the customers. These actions were validated by the criteria of experts previously selected using the Delphi method. The implementation of the actions to impact the qualification level, contributed to increase the know-how of the culinary specialists in traditional Cuban cuisine as well as the satisfaction of the clients at the Hotel Colonial Cayo Coco. These results constitute a feasible instrument for quality management of food services in tourist facilities throughout the country.

Keywords: traditional Cuban cuisine, professional qualification, tourism.

\section{Introducción}

En la actualidad el turismo es reconocido como una de las actividades más dinámicas de la economía global. Como categoría mundial de exportación este sector ocupa el tercer puesto, por detrás de combustibles y de productos químicos, y por delante de alimentación y de la industria de automoción. En muchos países en desarrollo, el turismo es incluso el primer sector en exportaciones (López, 2016).

Pero es en las localidades donde se instaura un espacio propicio para el intercambio de creencias, conocimientos y de prácticas heredadas $y / 0$ aprendidas que son compartidas por la comunidad como parte de la oferta turística propia de una cultura (Meléndez y Cañez, 2010).

La cocina tradicional local constituye un recurso turístico que ha motivado el traslado de millones de personas anualmente a diversas partes del mundo, con el objetivo de descubrir nuevos placeres sensoriales y vivir gratas experiencias culinarias (Bahls, Wedhausen y Silva, 2019), además de conocer tradiciones, rituales y costumbres alimentarias de los pueblos que visitan.

La cocina cubana proviene de la fusión de las cocinas española, árabe, africana, china, italiana y musulmana, que fue instaurándose a la luz de los acontecimientos históricos y sociales que se desencadenaron durante varios siglos (Calgaro y Trucco, 2007). Es en la cocina tradicional cubana donde se concretan los saberes culinarios, las costumbres y los rituales, así como las formas de elaboración de los alimentos reconocidas y transmitidas de generación en generación, que permanecen como parte de la 
identidad cultural, demandada por los turistas que visitan el país y reconocida a nivel internacional.

De acuerdo con Meléndez y Cañez, (2010), se identifica a la cocina tradicional cubana como un recurso endógeno cuyo aprovechamiento presenta potencialidades para elevar la calidad de vida de la población. El fomento de la oferta de cocina tradicional cubana en el sector turístico del país, constituye una ventaja competitiva a partir del aprovechamiento de la experiencia colectiva y la especialización productiva que produce el efecto de distrito descrito por Marshall en los distritos industriales (Vila, 2019). El incremento de la competitividad de los productos de la cocina tradicional cubana representa un factor susceptible para fortalecer la gestión del desarrollo local en los municipios cubanos, a través de una certera planificación del gobierno local.

Sin embargo, en estudios realizados por investigadores cubanos sobre la oferta culinaria de cocina tradicional cubana en instalaciones turísticas del país (Espinosa, Romaní, Hernández y Arias, 2019; López, 2016), se han identificado limitaciones que generaron insatisfacciones de los clientes con este servicio. Las insatisfacciones están referidas fundamentalmente, al elevado contenido de grasa en el caso de los platos principales y de azúcar en los postres, y a una limitada variedad de la oferta.

Carballo, Blanco, Betancourt y Ramos (2016) en la consulta de las encuestas corporativas aplicadas en el Hotel Colonial Cayo Coco de la provincia de Ciego de Ávila, encontraron que el $90 \%$ de las quejas se refiere a la insatisfacción con la variedad y calidad de su oferta de cocina tradicional cubana. En este hotel, se realizó un diagnóstico fáctico, por los autores de este trabajo, con el objetivo de diagnosticar el nivel de conocimiento de sus especialistas culinarios en temáticas de cocina tradicional cubana. Se entiende como especialistas culinarios a los cocineros que poseen un amplio conocimiento y experiencia en las elaboraciones culinarias en el área de la cocina donde se desempeñe, así pueden ser cocineros especializados en salsa (chef saucier), parrillas (chef grillardin, pescados (poissonnier, repostería (chef patissier), etc. El diagnostico 
develó que los especialistas culinarios presentaron limitaciones en el conocimiento de las tendencias actuales de la gastronomía, así como en aplicación de técnicas de cocción y decoración de las presentaciones culinarias. Por tal motivo, este trabajo tuvo como objetivo mostrar los principales resultados en la implementación de acciones para la preparación en cocina tradicional cubana de los especialistas culinarios del Hotel Colonial Cayo Coco.

\section{Materiales y métodos}

El estudio tiene naturaleza descriptiva-explicativa, se realizó mediante un estudio de caso para lo cual se seleccionó como objeto de estudio, el Hotel Colonial Cayo Coco ubicado en el polo turístico Jardines del Rey de la provincia de Ciego de Ávila, en la región central de Cuba. Se emplearon métodos de investigación del nivel teórico y empírico, como el análisis-síntesis, la inducción-deducción, la medición, la observación y la encuesta (Ramos, 2016). La observación participante se realizó durante los meses de enero a marzo, etapas en las que se registró la mayor afluencia de clientes al hotel.

El procedimiento metodológico se estructuró en cuatro etapas según se explica a continuación:

Etapa I. Caracterización del servicio de alimentación del Hotel Colonial Cayo Coco

Esta primera etapa se realizó con el objetivo de identificar las potencialidades que tiene el hotel para ofertar platos tradicionales cubanos. Para ello se realizó una revisión documental donde se consultaron las páginas web del Grupo Hotelero Cubanacan S.A y del Hotel objeto de estudio, así como su manual de gestión.

Etapa II. Evaluación de la satisfacción de los clientes con la calidad de la oferta de cocina tradicional cubana.

Se realizó con el objetivo de identificar los requerimientos de preparación de los especialistas culinarios mediante las insuficiencias manifiestas en la calidad del servicio de alimentación percibida por los clientes. Para ello, se aplicó una encuesta dirigida a los clientes del hotel con el objetivo de evaluar su 
satisfacción en cuanto a la calidad de la oferta culinaria de cocina tradicional cubana. Se evaluaron 5 atributos, adaptados de Espinosa et al., (2019), estos fueron: presentación, variedad, sabor, temperatura y tamaño de la ración. Se solicitó a cada encuestado su criterio de evaluación para cada atributo seleccionado de acuerdo con una escala numérica del 1 al 5 , siendo: 1 totalmente insatisfecho, 2 insatisfecho, 3 ni insatisfecho ni satisfecho, 4 satisfecho, y 5 totalmente satisfecho. Esta escala numérica se fundamentó con el instrumento de medición escala Likert de evaluación impar. A los encuestados se les indicó una pregunta abierta para que emitieran comentarios.

El procesamiento estadístico se realizó mediante el cálculo del promedio de la puntuación obtenida en cada atributo evaluado. Luego, se halló el porcentaje que representa el promedio de la puntuación obtenida en cada atributo evaluado con relación a la puntuación máxima (5 puntos) de la escala Likert propuesta. A partir de los valores obtenidos en cada atributo evaluado, se halló un promedio general que representó la evaluación de la satisfacción general de los clientes con la oferta culinaria de cocina tradicional cubana.

La muestra estadística para encuestar se seleccionó mediante un procedimiento probabilístico, a través de un muestreo aleatorio simple. A partir de una población de 282 clientes, se determinó el tamaño de la muestra con el empleo de la fórmula para poblaciones finitas (Morillas, 2007), y se consideró un margen de error del $10 \%$ y un nivel de confianza del 99,5\%. En este $\begin{array}{lll}\text { estudio se realizaron } 147 & \end{array}$ observaciones, excediendo el tamaño muestral calculado inicialmente.

Etapa III. Elaboración de acciones para la preparación en cocina tradicional cubana de los especialistas culinarios del Hotel Colonial Cayo Coco.

Se elaboró una propuesta de acciones de preparación mediante el trabajo en grupo y la aplicación de la técnica de tormenta de ideas (Tejeda, 2004), con la participación de especialistas y directivos del departamento de gestión de alimentos y bebidas del hotel y 
profesores del Departamento de Ciencia y Tecnología de los Alimentos de la Universidad de Camagüey Ignacio Agramonte Loynaz.

Las acciones de preparación propuestas fueron validadas mediante la consulta a expertos. La selección de expertos se realizó mediante el método de Delphi según Cabero e Infante (2014). Los candidatos a expertos se seleccionaron atendiendo a los criterios siguientes: 1) Estar dispuesto a participar en condición de expertos y 2) Tener una experiencia acumulada relacionada con los servicios de alimentación en el sector turístico cubano, no inferior a 10 años, ya sea desde el punto de vista académico, investigativo 0 profesional. Se evaluó el nivel de competencia de los candidatos a expertos y fueron seleccionados como expertos para este estudio los que obtuvieron un coeficiente de competencia alto $(K \geq 0,80)$.

Luego, se solicitó a los expertos su valoración sobre la propuesta de acciones para la preparación en cocina tradicional cubana de los especialistas culinarios del Hotel Colonial Cayo Coco. A partir de los criterios de evaluación previamente informados, los expertos emitieron juicio en una escala con un rango de respuesta de uno (1) a 10, siendo uno (1) la menor manifestación de cada criterio y 10 la mayor manifestación. El grado de concordancia entre los expertos se evaluó a través del coeficiente de concordancia de Kendall con el uso del software SPSS versión.22.

\section{Etapa IV. Aplicación y evaluación de las acciones de preparación.}

Las acciones de preparación se implementaron en cuatro pasos: sensibilización y diagnóstico; planeación; ejecución y evaluación. Se evaluó la factibilidad de la introducción parcial, a los cinco meses de avanzada la preparación. Para ello, se aplicó de la encuesta empleada en este trabajo para la evaluación de la satisfacción de los clientes con la calidad de la oferta de cocina tradicional cubana y los resultados se compararon con los obtenidos antes de la aplicación de las acciones de preparación. En este caso se encuestaron a 50 clientes mediante un muestreo de tipo intencional. 


\section{Resultados}

Caracterización del servicio de alimentación en el Hotel Colonial Cayo Coco

El Hotel Colonial Cayo Coco está situado en una de las zonas ecológicas más importantes del país. La instalación fue inaugurada en 1993 con el nombre Jardín de los Cocos y categoría de 5 estrellas, bajo la administración de la cadena española Guitar Hoteles. Después de varios años de cambios de nombres y cadenas hoteleras encargadas de su gestión, en junio de 2012 el hotel dejó de ser administrado por la cadena Blau y desde entonces pertenece al Grupo Cubanacán que opera como marca propia bajo la modalidad "todo incluido" con categoría de 4 estrellas. El principal mercado que visita el destino es el canadiense, que representa el $98 \%$ de los clientes, generalmente de la tercera edad, perteneciente fundamentalmente a la clase media-baja. La estancia promedio es de una semana; con predominio del viaje en familia (Carballo et al., 2016).

El servicio de alimentos y bebidas presenta varias tipologías e instalaciones entre los que se destacan: dos bufés, cuatro restaurantes especializados, dos parrilladas y siete bares. De ellos, se destaca el restaurante "El Caribeño" que es especializado la elaboración de comidas cubanas, además, el hotel ofrece una cena típica cubana una vez por semana. También constituye una potencialidad el hecho de contar, en el departamento de la cocina, con 20 especialistas culinarios, 4 mujeres y 16 hombres, todos graduados del curso de cocina del sistema de formación del turismo en Cuba (FORMATUR).

Evaluación de la satisfacción de los clientes con la calidad de la oferta de cocina tradicional cubana

Se encuestaron clientes de ambos sexos, con predominio del sexo femenino (58,3\%), la mayoría (52\%), se encontraban en el rango de edad de 40 a 65 años, procedentes de diversas nacionalidades, básicamente canadienses, estadounidenses, ingleses y españoles.

Los resultados obtenidos al evaluar los atributos que repercuten en la satisfacción de los clientes con la calidad de la oferta de cocina tradicional cubana en el hotel se 
exponen en la Figura 1. Se encontró que los atributos temperatura $y$ tamaño de la ración fueron evaluados por debajo del nivel de satisfacción percibido por los clientes, pues los valores cuantitativos obtenidos fueron inferiores al $60 \%$, de acuerdo con la escala Likert empleada. De ellos, se señala la variedad de la oferta sobre el cual se obtuvieron criterios de insatisfacción.

Figura 1. Satisfacción de los clientes con los atributos que repercuten en la calidad de la oferta de cocina tradicional cubana en el Hotel Colonial Cayo Coco.
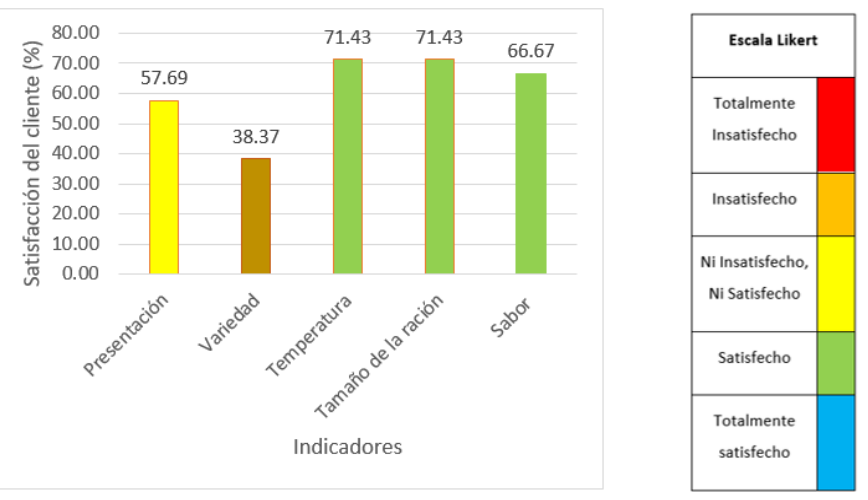

Fuente: Elaboración de los autores (2020).

Los comentarios emitidos sobre las insatisfacciones con la variedad de la oferta de cocina tradicional cubana estuvieron referidos a:

- Menú limitado en productos del mar.

- Escasez de frutas y vegetales frescos y de estación.

- Ausencia de jugos de frutas típicas del país.
- Predominio de carnes de cerdo y pollo como plato principal, realizando su cocción habitualmente mediante el método de asado.

- Se limita la oferta de arroz, a la elaboración de arroz moros y cristianos.

- -Carencia de variedad de quesos nacionales, enmarcados solamente al queso blanco.

- Carencia de variedad en los postres tradicionales cubanos, restringiendo la oferta a dulces en conserva.

Los resultados obtenidos en este estudio concuerdan con los reportados por Espinosa et al (2019) quienes encontraron que la variedad de las elaboraciones fue el atributo más devaluado por los turistas tanto en instalaciones estatales como privadas, en La Habana.

Esta insatisfacción no solo es causada por la escasez de alimentos en el menú que pudiera estar condicionada por factores de tipo productivo (estacionalidad), de gestión, entre otros; sino que se evidencia una insuficiente creatividad en la confección de los menús y desconocimiento de la rica variedad de platos tradicionales 
cubanos. Estas insuficiencias identificadas son susceptibles a su corrección mediante la preparación del recurso humano que labora en la cocina.

La insatisfacción con el atributo presentación, estuvo dada principalmente con la no aplicación de las tendencias actuales de la restauración sobre el ensamblado de los platos, la decoración, el contraste de texturas y colores en las ofertas. Además, los clientes se quejaron con la presencia de grasas de origen animal y vegetal en proporciones significativas en gran parte de los platos culinarios. Teniendo en cuenta la relación que hay entre el contenido de grasa en las elaboraciones culinarias y su sabor, se infiere que sea una de las causas que incidió de forma negativa en el porcentaje de satisfacción percibido por los clientes respecto al atributo sabor. Estos comentarios ratifican el criterio relacionado con el desconocimiento y/o no aplicación de las tendencias actuales de la restauración, como lo es la estilización de la cocina. Estilizar la cocina tradicional cubana no solo se refiere a la forma artística, creadora y novedosa de presentar los platos, sino que se deben tener en cuenta las normas dietéticas en las elaboraciones culinarias (uso racional del azúcar, sal, grasas, etc.), preservando la autenticidad del plato (Fernández y Gutiérrez, 2016).

La satisfacción de los clientes respecto a los atributos temperatura y tamaño de la ración obtenida en este trabajo, coincide con lo reportado por Espinosa et al, (2019).

De manera general, se constató que la calidad de las elaboraciones de cocina tradicional cubana ofertada en el Hotel Colonial Cayo Coco satisfizo a los turistas encuestados, ver la figura 2.

Figura 2. Evaluación general de la satisfacción del cliente con la oferta de cocina tradicional cubana en el Hotel Colonial Cayo Coco.

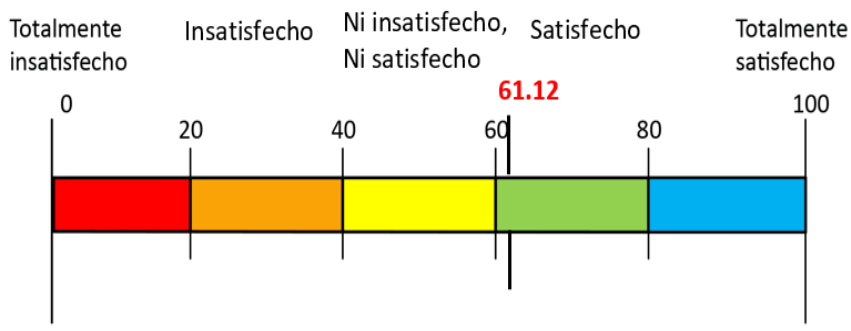

Fuente: Elaboración de los autores (2020).

Sin embargo, se debe destacar que el porcentaje obtenido (61.12\%) está próximo al límite mínimo (60\%) del nivel de satisfacción establecido en la escala Likert empleada. 


\section{Elaboración de las acciones de preparación}

Las insatisfacciones con la calidad de la oferta de cocina tradicional cubana identificadas en etapas anteriores, guardan una estrecha relación con los niveles de preparación de los especialistas culinarios del Hotel Colonial Cayo Coco y demandan acciones para su superación de este profesional que permitan el mejoramiento de su desempeño.

En este sentido, se asume la preparación como una vía de superación continua, que parte de las necesidades y potencialidades de los profesionales y sus contextos de actuación, y está necesariamente incorporada a la práctica profesional, centrada en la apropiación y mejoramiento del modo de actuación profesional (García, Portuondo y Guzmán, 2017, p. 379).

A partir de la definición de preparación asumida, se identificaron los atributos fundamentales que se tuvieron en cuenta en el diseño de las acciones de preparación de los profesionales de la cocina del Hotel Colonial Cayo Coco, que se resumen como:
- Se diseñan a partir de las necesidades manifiestas de preparación de los especialistas culinarios que inciden en la satisfacción de los clientes con la calidad de la oferta de cocina tradicional cubana en el hotel.

- Tienen el propósito de actualizar y perfeccionar los conocimientos $y$ habilidades en cocina tradicional cubana de los especialistas culinarios del hotel. - Contribuyen a la preparación de este profesional a partir de un proceso formativo continuo, planificado, sistemático, integral y contextualizado.

Como resultado del trabajo en grupo y la aplicación de la técnica de tormenta de ideas se propusieron acciones para la preparación del personal que labora en la cocina del Hotel Colonial Cayo Coco. Estas acciones fueron sometidas a la validación a través del criterio de expertos, previamente seleccionados y comprobada su competencia según el procedimiento referenciado en este trabajo.

Como posibles expertos se identificaron a 18 personas, pero se les aplicó el instrumento de selección solo a 13 ya que cinco no dieron la 
disponibilidad. Los resultados obtenidos en la encuesta mostraron que las 13 personas obtuvieron un coeficiente de competencia con valores comprendidos en el rango de 0.81 a 1, clasificándose como expertos de alta competencia. Por tanto, se determinó consultar a los expertos seleccionados para obtener su criterio para la validación de las acciones de preparación propuestas en este trabajo, teniendo en cuenta los indicadores siguientes:

- Nivel de correspondencia entre las necesidades manifiestas de preparación de los especialistas culinarios y los conocimientos $y$ habilidades profesionales que se persiguen actualizar $y$ perfeccionar.

- Nivel de orientación a la solución de los problemas diagnosticados en el contexto de actuación de los especialistas culinarios al que está dirigida la preparación mediante los resultados esperados de las acciones propuestas.

- Nivel de correspondencia entre las formas organizativas de preparación planificadas y su orientación al mejoramiento del desempeño de los especialistas culinarios, en el propio contexto de su actividad laboral.

- Organización y secuencia lógica de las acciones propuestas.

Además, se presentó una pregunta abierta con el propósito de obtener el criterio de los expertos sobre la posibilidad de eliminarse $o$ añadirse acciones de preparación en función de mejorar la factibilidad su aplicación.

Tras realizarse dos rondas de la técnica Delphi se comprobó el criterio de expertos sobre la factibilidad de las acciones propuestas, obteniéndose que la distribución Chi Cuadrado $\left(\mathrm{x}^{2}\right)$ calculada $\left(x^{2}=140,751\right)$ es mayor que la teórica, por lo que se rechaza hipótesis nula $(p=0)$ y se acepta hipótesis alternativa $\quad(p>0)$, concluyendo que el coeficiente de Kendall $(\mathrm{W})$ obtenido $(\mathrm{W}=0,833)$ es significativamente distinto de cero lo que indica que existe concordancia entre los criterios de los expertos. De esta forma quedaron establecidas las acciones de preparación de los especialistas culinarios del Hotel Colonial Cayo Coco.

Aplicación y evaluación de las acciones de preparación. 
La aplicación de las acciones de preparación se realizó en cuatro pasos: sensibilización y diagnóstico; planeación; ejecución y evaluación. A continuación, se explican las tareas desarrolladas en cada paso.

Paso I: Sensibilización y diagnóstico.

En este paso se realizaron reuniones con la dirección del hotel y para sensibilizarlos sobre la necesidad de preparación en cocina tradicional cubana de los especialistas culinarios y propiciar así su disposición y compromiso con el proceso. Además, se socializaron las potencialidades y carencias que manifestaron los especialistas culinarios en relación con los contenidos teóricos, metodológicos y prácticos que constituyen objeto de la preparación, con el fin definir de forma consensuada los problemas a resolver.

\section{Paso II: Diseño y planeación.}

En este paso se planificaron las acciones de preparación, teniendo en cuenta las potencialidades del hotel, para alcanzar los objetivos de la preparación. Se determinaron los lugares, horarios, los recursos materiales y humanos para el desarrollo de las actividades de preparación. Se elaboraron los programas de las acciones de preparación, a partir de las formas organizativas seleccionadas, el curso y el entrenamiento. El curso para contribuir a la preparación de los especialistas culinarios, en función de la actualización de conocimientos de las nuevas tendencias en el campo de la ciencia y la tecnología de cocina, y el entrenamiento para demostrar los conocimientos, habilidades y valores profesionales en el desempeño de su actividad laboral.

En la elaboración de los programas del curso y el entrenamiento de los especialistas culinarios se consideraron las insuficiencias reveladas en el diagnóstico inicial, los contenidos requeridos para cubrir las brechas en la preparación y los métodos y técnicas para la orientación de los contenidos. A continuación, se presentan los planes temáticos:

La evaluación tuvo un carácter sistemático, a través de la participación en las actividades presenciales, las tareas de investigación, las autoevaluaciones realizadas. Al finalizar el entrenamiento, se valoró la 
efectividad de los programas y de las actividades metodológicas, a partir de los criterios de los especialistas culinarios que reciben la preparación, así como de los docentes y directivos del hotel, en función del mejoramiento de esta.

Tabla 1. Plan temático del curso de preparación en cocina tradicional cubana.

\begin{tabular}{|l|l|l|}
\hline Temas & Plan temático & $\begin{array}{l}\text { Horas } \\
\text { lectivas }\end{array}$ \\
\hline I & Confección de un menú temático de cocina tradicional cubana. & 16 \\
\hline II & $\begin{array}{l}\text { Aplicación de las tendencias y técnicas modernas de la gastronomía } \\
\text { para la estilización de los platos tradicionales cubanos. }\end{array}$ & 16 \\
\hline III & $\begin{array}{l}\text { Aplicación de las medidas de seguridad e higiene en la manipulación } \\
\text { de los alimentos. }\end{array}$ & 16 \\
\hline Total de horas de actividad presencial & 48 \\
\hline Total de horas de actividad práctica & 92 \\
\hline
\end{tabular}

Fuente: Elaboración de los autores (2020)

Tabla 2. Plan temático de entrenamiento en cocina tradicional cubana.

\begin{tabular}{|l|l|l|l|}
\hline Temas & \multicolumn{1}{|c|}{ Plan temático } & \multicolumn{1}{|c|}{$\begin{array}{c}\text { Horas } \\
\text { lectivas }\end{array}$} & $\begin{array}{c}\text { Horas no } \\
\text { presenciales }\end{array}$ \\
\hline I & $\begin{array}{l}\text { La cocina cubana como elemento de identidad } \\
\text { cultural, su origen y evolución. }\end{array}$ & 8 & 14 \\
\hline II & $\begin{array}{l}\text { Tendencias actuales de la gastronomía. La } \\
\text { estilización de la cocina tradicional cubana. }\end{array}$ & 6 & 18 \\
\hline III & $\begin{array}{l}\text { Las técnicas modernas de cocción de los alimentos: } \\
\text { sabor, ciencia y arte. }\end{array}$ & 8 & 16 \\
\hline IV & $\begin{array}{l}\text { La inocuidad de los alimentos en las elaboraciones } \\
\text { culinarias. }\end{array}$ & 6 & 20 \\
\hline V & $\begin{array}{l}\text { Los platos tradicionales cubanos como atractivo } \\
\text { turístico en la oferta gastronómica hotelera. }\end{array}$ & 6 & 16 \\
\hline \multicolumn{2}{|l|}{ Total de horas del curso } & 34 & 84 \\
\hline
\end{tabular}

Fuente: Elaboración de los autores (2020)

Paso III: Ejecución

Se implementaron las acciones de preparación en cocina tradicional cubana de los especialistas culinarios del Hotel Colonial Cayo Coco con una duración planificada de ocho meses. Las acciones se realizaron de acuerdo con lo planificado en cuanto a las fechas, 
lugares, y participantes (especialistas culinarios y los profesores facilitadores de las actividades). Durante la ejecución de las actividades de preparación se llevó a cabo el control sistemático y la evaluación de los contenidos según el plan coordinado.

\section{Paso IV: Evaluación}

Esta etapa tuvo como objetivo valorar los resultados de las acciones aplicadas en la práctica y el nivel de preparación en cocina tradicional cubana alcanzado por especialistas culinarios del Hotel Colonial Cayo Coco.

Con respecto a la exploración empírica de la factibilidad de las acciones de preparación, se puede inferir que dicha introducción parcial ha mostrado hasta el momento en que se realiza este trabajo, resultados satisfactorios. Considerando el avance de los 5 primeros meses de desarrollo de las acciones de preparación planificadas, se constató mediante la observación en los eventos de alimentación que se ofertan en el hotel: mayor variedad de platos tradicionales cubanos, perfeccionada la decoración de los platos en su presentación, así como la incorporación de nuevas técnicas de cocción de los alimentos.

Los resultados obtenidos en la encuesta de evaluación de la satisfacción de los clientes con la calidad de la oferta de cocina tradicional cubana aplicada después de la ejecución parcial de las acciones de preparación y su comparación con los obtenidos antes, se muestran en la figura 3. Los resultados demuestran que la preparación de los especialistas no solo influyó en la mejora en los atributos que no satisficieron a los clientes en el diagnóstico inicial (presentación y variedad) sino que hubo un incremento con la satisfacción de calidad total de la oferta de cocina tradicional cubana (69.09 \% de satisfacción).

Figura. 3. Nivel de satisfacción de los clientes con la calidad de los platos tradicionales cubanos aplicados antes $y$ después de las acciones de preparación.

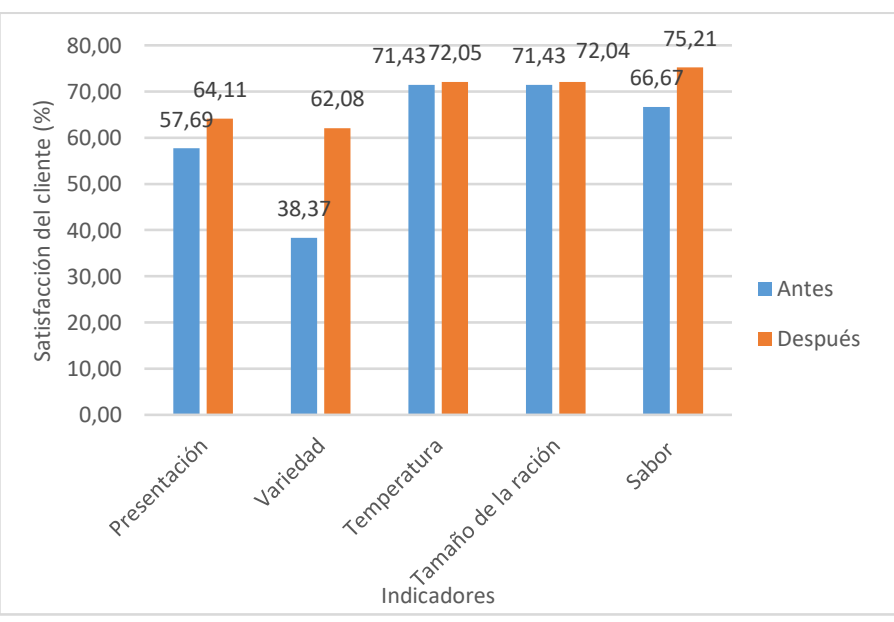

Fuente: Elaboración de los autores (2020) 


\section{Conclusiones}

Los resultados obtenidos mediante la aplicación de diferentes métodos de investigación permitieron comprobar que existían dificultades en la preparación de los especialistas culinarios del Hotel Colonial Cayo Coco, lo que generaba insatisfacciones de los clientes con la oferta de cocina tradicional cubana.

Los resultados evidenciados con la implementación de las acciones de preparación revelaron un aumento en la satisfacción de los clientes del Hotel Colonial Cayo Coco, mediante el mejoramiento de la preparación de los especialistas culinarios en temáticas de cocina tradicional cubana.

Las acciones de preparación en cocina tradicional cubana de los especialistas culinarios del hotel constituyen un instrumento que permite el diagnóstico, planificación, ejecución y evaluación de este proceso en la práctica, por lo que ofrece una referencia factible para la gestión de la calidad de los servicios de alimentación en instalaciones turísticas del país.

\section{Bibliografía}

Bahls, A., Krause, R., \& da Silva, E. (2019). Comprensión de los conceptos de culinaria y gastronomía: una revisión y propuesta conceptual. Estudios y perspectivas en turismo, 28(2), 312-330. http://www.estudiosenturismo .com.ar/PDF/V28/N02/v28n2 a04.pdf

Cabero, J., \& Infante, A. (2014). Empleo del método Delphi y su empleo en la investigación en comunicación y educación. EDUTEC. Revista electrónica de tecnología educativa, (48), a272-a272.

http://edutec.rediris.es/Revele c2/Revelec48/n48_Cabero_In fante.html

Calgaro, S., \& Trucco, M. (2007). La cocina cubana, sabores y tradiciones. https://repotur.yvera.tur.ar/bit stream/handle/123456789/44 64/

Carballo, E., Blanco, K., Betancourt, M., \& Ramos, E. (2016). La innovación de producto en la formación de la imagen percibida. Caso Hotel Colonial Cayo Coco, destino turístico Jardines del Rey, Cuba. Retos de la Dirección, 10(1), 114141. S230691552016000100008

Espinosa, J., Romaní, B., Hernández, L., \& Arias, R. (2017). Contribución de la 
gastronomía típica al desarrollo turístico del destino Cuba. Explorador Digital, 1(3), 28-38.

https://doi.org/10.33262/explo radordigital.v1i1.313

Fernández, E. y Gutiérrez, S. (2016). El sabor de la cocina cubana. Santiago de Cuba, Cuba: Editorial Oriente, Instituto Cubano del Libro. ISBN 978959-11-1026-8.

García, A., Portuondo, R., \& Guzmán, R. (2017). El proceso de preparación de los supervisores de las escuelas técnicas.

TRANSFORMACIÓN. 13 (3), 374-385. S207729552017000300005

López, D. (2016). Propuesta de mejora al diseño del menú del restaurante Internacional del hotel Brisas Guardalavaca (Bachelor's thesis, Universidad de Holguín Oscar Lucero Moya, Facultad de Ciencias Empresariales y Administración, Departamento de Turismo). https://repositorio.uho.edu.cu/ jspui/bitstream/uho/4814/1/

Meléndez Torres, J., \& Cañez, M. (2010). La cocina tradicional regional como un elemento de identidad y desarrollo local: el caso de San Pedro El Saucito, Sonora, México. Estudios sociales (Hermosillo, Son.), 17(SPE), 181-204. v17nspe/v17nspea8.pdf
Morillas, A. (2007). Muestreo en poblaciones finitas. Obtenido de http://webpersonal. uma. es/ morillas/muestreo. pdf, 1 30. http://webpersonal. uma. es/ morillas/muestreo. pdf, 1 30.

Ramos, E. (2016). Métodos y técnicas de investigación. Obtenido de Gestiopolis: https://www. gestiopolis. com/metodos-y-tecnicas-deinvestigacion. http://www.gestiopolis.com/m etodos-y-tecnicas-de-investig acion/

Tejada, J. (2004). La intervención en grupos. Técnicas de trabajo: CIFO.

https://www.yumpu.com/es/d ocument/view/14817556/4

Vila, J. (2019). Distritos industriales y renta económica: el efecto distrito-renta. Papeles de Europa, 32(1), 67-88. https://revistas.ucm.es/index. php/PADE/article/view/64472 